\title{
Efficacy, tolerability, quality of life and spasticity in outpatients with remitting multiple sclerosis (RMS) treated with glatiramer acetate (GA) - Results of an observational study
}

\author{
Haupts $\mathrm{M}^{1 *}$, Klasser $\mathrm{M}^{2}$ and Faude $\mathrm{U}^{3}$ \\ ${ }^{1}$ Augustahospital Anholt Department of Neurology Augustastrasse 8, 46419 Isselburg-Anholt, Germany \\ ${ }^{2}$ gwd consult $\cdot$ contract research Mozartstrasse 2-4 63165 Muehlheim am Main, Germany \\ ${ }^{3}$ TEVA GmbH Charlottenstraße 5910117 Berlin, Germany
}

\begin{abstract}
Background: The aim of this project was to observe spasticity and its possible improvement using the new standardized multiple sclerosis spasticity scale (MSSS-88) to compare with other instruments for assessment of burden of disease in patients treated with Glatiramer acetate (Copaxone ${ }^{\circledR}$ ), an approved disease-modifying agent consisting in millions of different polypeptides and amino acids.

Methods: 305 patients suffering from remitting multiple sclerosis (RMS) with spasticity symptoms grade 1 according to Ashworth scale were treated with at least $20 \mathrm{mg} / \mathrm{ml} \mathrm{GA}$ daily and observed for one year. Clinical parameters assesed besides MSSS-88 were: Relapse rate, Expanded Disablity Status Scale (EDSS), Modified Ashworth Scale (MAS), EuroQol Five Dimensions Questionnaire (EQ-5D) and magnetic resonance imaging (MRI).

Results: Over one year annualized relapse rate and EDSS remained stable. Only $13.6 \%$ of the patients showed a progression of the disease. The Ashworth Spasticity Score and MSSS-88 total score remained rather unchanged, whereby best improvements were observed for muscle stiffness (41.08\%), emotional health (46.47\%) and pain and discomfort (41.91\%). The patient's health status remained stable or improved $>80 \%$ in nearly all dimensions of EQ-5D. In 33 pairs of MRI scans, a majority $63.6 \%$ was classified as "stable" or "improved" after one year, compared to only $24.2 \%$ at the beginning of the study.

Conclusion: A representative sample of remitting multiple sclerosis (RMS) patients was assessed during daily practice conditions. Results indicate the potential of immune modulating therapy to stabilize progression of disease measured by more conventional instruments as ARR and EDSS, but also to keep stable the progression of spasticity symptoms as assessed by established and new scales. Freedom from clinical disease activity was reached in $56 \%$ of the patients during the year of the study. Spasticity did not progress. Patient reported outcomes (PRO) proved suitable for assessment: the Multiple sclerosis spasticity scale (MSSS-88) and the EuroQuol Questionnaire EQ-5D yielded consistent results with significant correlations. Tolerability of the drug was very good or good in $>90 \%$ of the patients. Future investigational activities would be helpful to further explain mechanisms of action of Copaxone ${ }^{\circledR}$ on immunological functions, and neurological pathomechanisms of the development of spasticity in MS patients.
\end{abstract}

\section{Introduction}

Spasticity is a shared feature in many neurological conditions including multiple sclerosis (MS), cerebral ischemia and spinal cord injury. Epidemiological studies indicate that spasticity is a significant contributor to disability and reduced quality of life in up to $80 \%$ of patients with MS [1-3]. Spasticity starts in from of painful cramps, progresses to spasms or transient cloni and finally to continuous stiffness. GA is indicated for treatment of relapsing-remitting MS (RRMS) that does not seem to be connected with worsening of spasticity [4].

Glatiramer acetate (Copaxone ${ }^{\oplus}$ ), as a member of the class of non-biological complex drugs (NBCD), is used in patients suffering from remitting-relapsing MS for more than 20 years [5]. Unique characteristic of glatiramer acetate (GA) is the complexity of several millions of distinct polypeptides, which cannot be individually distinguished [6]. The enormous heterogeneity GA consists of about 1029 different amino acids. This is thought to trigger a shift of derailed immune system in MS patients resulting in therapeutic effects shown in non-progressive stages of the disease, mostly demonstrated as reduction of annualized relapse rate in controlled clinical trials or observational protocols. Driven by the development of more feasible scores in clinical routine, spasticity is addressed in remitting-relapsing multiple sclerosis (RRMS) patients more recently, because of the strong impact on burden of disease for patients concerned [7]. Former clinical investigations showed spasticity improvements in RRMS patients switched from interferon- $\beta$ to GA [8]. The aim of this project

Correspondence to: Haupts $M$, Augustahospital Anholt, Department of Neurology, Augustastrasse 8, 46419 Isselburg-Anholt, Germany, E-mail: Michael.Haupts@ruhr-uni-bochum.de

Key words: multiple sclerosis, spasticity, glatiramer acetate, NEDA, patientreported outcomes (PRO), quality of life

Received: February 28, 2018; Accepted: March 15, 2018; Published: March 19, 2018 
Haupts M (2018) Efficacy, tolerability, quality of life and spasticity in outpatients with remitting multiple sclerosis (RMS) treated with glatiramer acetate (GA) Results of an observational study

was to observe spasticity and its possible improvement using the new standardized multiple sclerosis spasticity scale (MSSS-88) to compare with other instruments for assessment of burden of disease in patients treated with GA.

\section{Methods/Patients}

\section{Study design and patients}

This non-interventional observational study was conducted between January 2014 and June 2015 in Germany in 89 centers. 305 Patients suffering from RRMS with spasticity symptoms grade 1 according to Ashworth scale were enrolled with given informed consent and received at least GA $20 \mathrm{mg} / \mathrm{ml}$ daily according to the decision of the physician. Patients were observed at baseline, month 1 , month 3 , month 6, month 9 and month 12 (final assessment).

The age of the patients ranged from 18 to 69 years (mean age: 41.2 years \pm 11.1 years standard deviation). Most patients were female (64.9\%). The median duration of MS was 14.8 years. Predominant type of MS was relapsing remitting (RRMS) with $89.5 \%$, according to the recent staging provided by the European Medicine Agency (EMA) the type of MS for these patients would be classified as remitting multiple sclerosis (RMS) (13). 7.4\% of enrolled patients suffered from CIS (clinically isolated syndrome) so these patients didn't fulfil the revised criteria by McDonald 2010 by magnetic resonance tomography (14) $68.1 \%$ of the patients were, de novo" patients. $26.3 \%$ switched to GA from interferone treatment. Main reasons for switching were: side effects of the previous medication (62.5\%), lack of efficacy of the previous medication (29.7\%) and/or progression of the disease (13.9\%). If pretreated with GA, the duration of pretreatment was between 7 days and 3 months in $75.3 \%$ of these cases.

Concomittant medication known to act on spasticity wa rare in this sample: Only 5,9\% were taking antispastic drugs, including gabapentin with such a known effect. This percentage remained stable throughout the study.

Table 1 is providing more details about the baseline characteristics of the study patients.

\section{Outcome measures}

Clinical parameters measured were

\section{Annualized relapse rate}

\section{EDSS (Expanded Disability Status Scale)}

The 19-point Kurtzke Expanded Disability Status Scale (EDSS) is the most widely accepted clinical disability scale [9]. It assigns a severity score to the patient's clinical status that ranges from $0-10$ in increments of 0.5 .

\section{MAS (Modified Ashworth Scale)}

A quick and easy way to measure spasticity. The MAS measures resistance during passive soft-tissue stretching [10].

\section{MSSS-88 (Multiple Sclerosis Spasticity Scale)}

The 88 -item scale attempts to quantify the impact of spasticity in eight clinically relevant areas: three spasticity specific symptoms (muscle stiffness, pain and discomfort and muscle spasms), three areas of physical functioning (ADL, walking, body movements), emotional health and social functioning [7].
Table 1. Baseline characteristics of the patients

\begin{tabular}{|l|l|}
\hline Number of patients (n1) from (n2) centres & $305 / 69$ \\
\hline Age (years; mean \pm SD) & $41.2( \pm 11.1)$ \\
\hline Gender (rel. freq) & female: 64.5\%: \\
\hline Duration of MS (years; median) & 14.8 \\
\hline Type of MS (McDonald's 2010 Criteria) & \\
- RRMS (thereof 62.8\% with incomplete remission) & $86.9 \%$ \\
- CIS & $7.2 \%$ \\
\hline No. of relapses in the last 3 years: & \\
none & $12.7 \%$ \\
1 & $37.1 \%$ \\
2 & $35.7 \%$ \\
3 & $8.9 \%$ \\
\hline$>3$ & $5.5 \%$ \\
Annualised relapse rate & $0.53( \pm 0.38)$ \\
\hline Staging of MS & \\
- stable (grade 1) & $30.0 \%$ \\
- rare exacerbations (grade 2a) & $41.6 \%$ \\
- slow progression (grade 2b) & $11.5 \%$ \\
- grade 3a and more & $16.9 \%$ \\
\hline Patient classification: & \\
- de-novo patient & $68.1 \%$ \\
- switcher patient from interferon therapy & $26.3 \%$ \\
\hline (Main) Reasons for switching: & \\
- side effects of the mediation & $65.2 \%$ \\
- lack of efficacy of the medication & $30.4 \%$ \\
- progression of the disease & $14.5 \%$ \\
\hline Duration of pre-treatemt with GA & $16.4 \%$ \\
- until 7 days & $75.3 \%$ \\
- > 7 day unteil 3 months & \\
\hline
\end{tabular}

\section{EQ-5D (EuroQol Five Dimensions Questionnaire)}

The EQ-5D is a brief, standardized, generic measure of generic health that provides a profile of patient function (dimensions: mobility, self-care, usual activities, pain/discomfort, anxiety/depression) and a global health state rating [11].

\section{MRI (Magnetic Resonance Imaging)}

Due to the noninterventional design of the study, MRI scans were done in a limited number of persons in local sites according to clinical routine without a formal protocol. Assessments and reports were done unblinded. Classified T2- lesion data were reported for 33 pairs of scans (at start and end of the study). There were typical multiple $(>5)$ lesions in $77.4 \%$ of scans initially.

Safety and tolerability of GA were assessed via the incidence of adverse drug reactions and a final global rating of the treating physician.

\section{Statistical analysis}

Outcome measures were analyzed using common descriptive statistical summaries. 95\% confidence intervals were computed if appropriate. Pre-post comparisons were made with Student's t-test (for dependent samples), Wilcoxon test (for rank scale data), and chisquare test (for nominal scale data). Due to the observational character of this study, no specific hypotheses were tested. Resulting p-values of the significance tests should be interpreted in an exploratory way, only.

\section{Results}

\section{Relapses}

During the observation period (median 11.2 months) the annualized relapse rate (ARR) did not change (baseline $0.53 \pm 0.38$, final assessment $0.54 \pm 1.43)$. The percentage of patients without relapse during the study improved significantly from $66.5 \%$ to $76.2 \%$ in the entire cohort $(n=248, p<0.0114)$, from $63.4 \%$ to $77.6 \%$ in de-novo 
Haupts M (2018) Efficacy, tolerability, quality of life and spasticity in outpatients with remitting multiple sclerosis (RMS) treated with glatiramer acetate (GA) Results of an observational study

patients $(\mathrm{n}=161, \mathrm{p}<0.0043)$ and not significantly from $67.2 \%$ to $71.6 \%$ $(\mathrm{n}=67, \mathrm{p}=0.5127)$ in patients who switched to GA therapy.

\section{Progression (EDSS)}

The EDSS score (mean \pm SD) remained stable in all patients $(\mathrm{n}=176)$ between baseline $(2.68 \pm 1.32)$, month $6(2.63 \pm 1.28)$ and final assessment $(2.67 \pm 1.30)$ Figures 1 and 2 .

\section{Combined criterion "No Evidence of Clinical Disease Activity (NEDA)"}

176 patients were evaluable for the clinical analysis of "no evidence of disease activity" (NEDA-2). 114 patients (64,77\%) didn't show either relapses or progression in EDSS during observation time in this noninterventional trial.

\section{MAS (Modified Ashworth Scale)}

The Ashworth spasticity score remained stable between baseline $1.2 \pm 0.4$ and the final assessment after 12 months $1.12 \pm 0.5$ Figure 3 Based on 229 patients, $21.8 \%$ showed an improvement, $66,4 \%$ remained stable and $11.8 \%$ of the patients experienced a worsening of symptoms.

\section{MSSS-88 (Multiple Sclerosis Spasticity Scale)}

MSSS- 88 was measured to assess severity and course of spasticity during observational period and did not change when calculated as total score at baseline vs. month $12(1.73 \pm 0.62$ vs. $1.72 \pm 0.62)$ including all 88 sub items Figure 4.

While $46 \%$ of the patients showed a deterioration of symptoms, $4.6 \%$ were stable and $46.9 \%$ improved. Sub items showing best improvements were muscle stiffness (41.08\%), emotional health

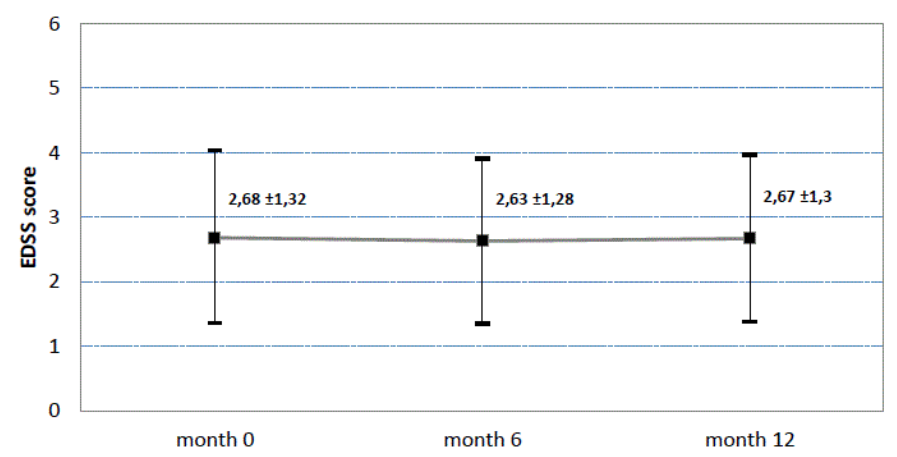

Figure 1. EDSS score at baseline, after 6 and after 12 months. Mean and standard deviation. $\mathrm{N}=176$. Last observation carried forward method for replacement of missing values.

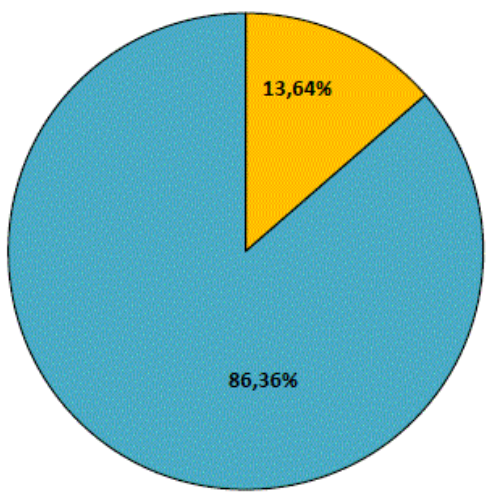

$\square$ progression

$\square$ no progression

Figure 2. Frequency of "progression" based on EDSS. n=176. Last observation carried forward method for replacement of missing values.

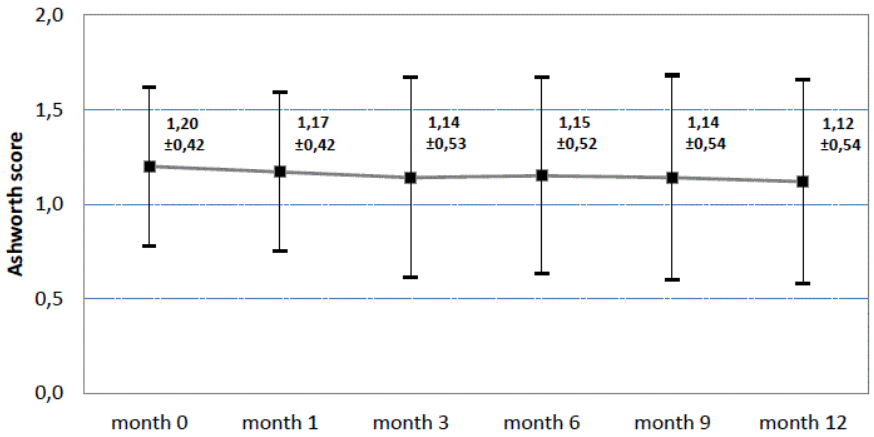

Figure 3. Asworth Score at baseline, after 1,3,6,9 and 12 months. Mean and standard deviation. $\mathrm{N}=299$. Last observation carried forward method for replacement of missing values.

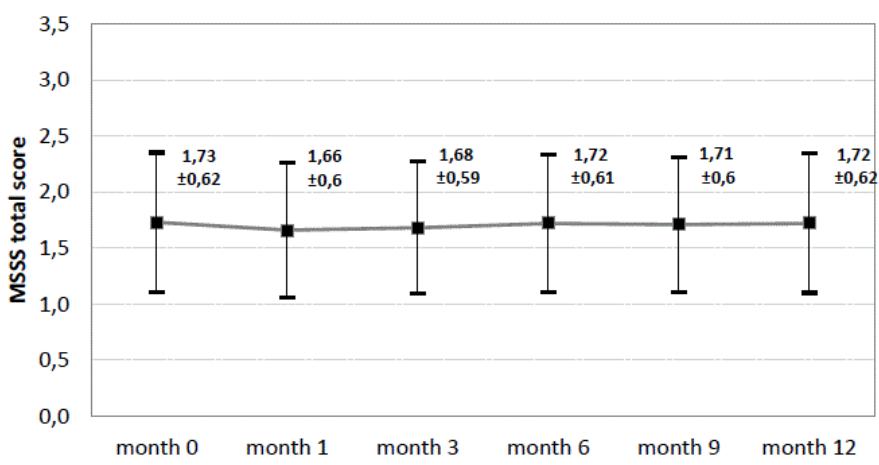

Figure 4. MSSS- 88 total score at baseline, after 1,3,6,9 and 12 moths. Mean and standard deviation. $n=241$. Last observation carried forward method for replacement of missing values.

(46.47\%) and pain and discomfort (41.91\%) Table 2. Neither changes in MSSS- 88 total score nor in MSSS- 88 subscales were statiscally signifcant.

The patient's health status remained stable or improved in all dimensions of EQ-5D: mobility $80.1 \%$, self-care $86.3 \%$, usual activities $78,4 \%$, pain/discomfort $78,8 \%$ and anxiety/depression $84.1 \%$. Best improvements were observed for pain/discomfort (29.7\%) and anxiety/ depression (26.6\%) (Figure 5).

The general health state estimate of EQ-5D on a 100 point visual analogue scale (VAS) remained stable over the course of time: $67.3 \pm$ 19.7 points (baseline) vs. $68.4 \pm 19.2$ points (month 12) (Figure 6).

In order to cross-validate the MSSS-88 Multiple Sclerosis Spasticity Scale we computed correlations with established MS scores and found statistically significant linear correlations with EQ-5D VAS (general health): $\mathrm{r}=0.61(\mathrm{p}<0.0001, \mathrm{n}=236)$ and with EDSS: $\mathrm{r}=0.46(\mathrm{p}=0.0001$, $\mathrm{n}=222$ ) (Figure 7).

\section{MRI findings}

MRI assessments were obtained for a limited number of persons only due to the noninterventional design of the study. In 33 pairs of scans, a majority $63.6 \%$ was classified as "stable" or improved after one year of this study by the local evaluating physicians, while initially only $24.2 \%$ had been categorized as "stable" and of $75,8 \%$ as "not stable/ active".

\section{Tolerability and safety}

At the final evaluation after twelve months physicians rated tolerability of GA in nearly $22 \%$ of the patients as ,very good“ $(32.3 \%)$ or "good“ (49.0\%). In a few cases it was rated, moderate $(12.3 \%)$ 
Haupts M (2018) Efficacy, tolerability, quality of life and spasticity in outpatients with remitting multiple sclerosis (RMS) treated with glatiramer acetate (GA) Results of an observational study

Table 2. Changes in MSSS- 88 sub items.

\begin{tabular}{|c|c|c|c|}
\hline & \multicolumn{3}{|c|}{ Proportion of patients (\%) } \\
\hline MSSS-88 item & improved & unchanged & worsened \\
\hline Muscle stiffness & $41.1 \%$ & $17.3 \%$ & $41.5 \%$ \\
\hline Emotional health & $46.5 \%$ & $20.3 \%$ & $33.2 \%$ \\
\hline Pain and discomfort & $41.9 \%$ & $18.7 \%$ & $39.4 \%$ \\
\hline Muscle spasms & $33.6 \%$ & $29.1 \%$ & $37.3 \%$ \\
\hline Activity of daily living & $32.8 \%$ & $33.2 \%$ & $34.0 \%$ \\
\hline Walking & $38.2 \%$ & $24.5 \%$ & $37.3 \%$ \\
\hline Body movements & $35.7 \%$ & $26.1 \%$ & $38.8 \%$ \\
\hline Social functioning & $36.1 \%$ & $24.9 \%$ & $39.0 \%$ \\
\hline
\end{tabular}

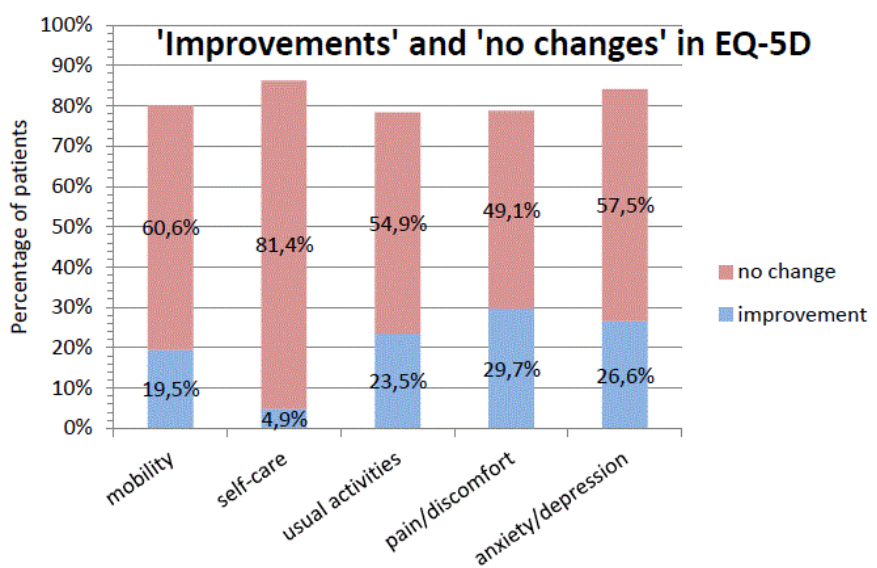

Figure 5. percentage pf patients with "improvements" or "no changes" in dimensions of EQ-5D. $n=226$.

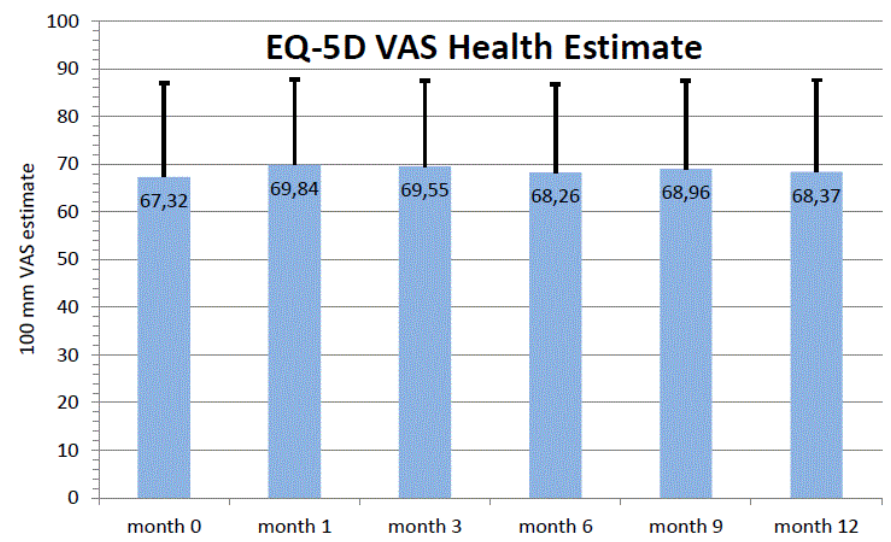

Figure 6. EQ-5D: VAS estimate of general health (mean and SD) during the study. $\mathrm{N}=225$. $0=$ worst health, $100=$ best health. Missing values have been imputed by LOCF.

or unsatisfactory (6.4\%). 77 patients $(25.3 \%)$ terminated the study prematurely. Patient's wish (49.4\%), lack of efficacy (19.5\%), and adverse events $(10.4 \%)$ were the most frequent reasons for premature termination. No case of progressive multifocal leukoencephalopathy (PML) was observed during the one year treatment period.

\section{Side effects}

A total of 30 out of 305 patients (9.8\%) suffered from 67 adverse events (AE) Table 3, mainly injection site reactions (35.8\%) or recurrence of MS (9\%). 60 out 67 AEs were considered to be causally related to the treatment with GA. Nine patients suffered from 25 serious adverse events (SAE). 19 of these 25 SAEs were probably related to GA.

\section{Discussion}

Non-interventional studies (NIS) allow observation and documentation of the efficacy, risks and side effects of a medical treatment in daily clinical practice. Compared to clinical trials with rather strict defined inclusion- and exclusion criteria, results of NIS provide high external validity due to the representativity of the population included. Due to the larger number of patients in NIS compared to controlled clinical trials the possibility for detection of

Table 3. Incidence of the most frequent adverse events of GA. Base value: $n=67$ AEs in 305 patients.

\begin{tabular}{|c|c|c|}
\hline Type of adverse event & $\mathbf{n}$ & $\%$ \\
\hline $\begin{array}{l}\text { Injection site reactions } \\
\text { - erythema at injecton site (4) } \\
\text { - swelling at injecton site (4) } \\
\text { - pain at injecton site (3) } \\
\text { - discomfort at injecton site (2) } \\
\text { - irritation of skin (2) } \\
\text { - skin reaction (1) } \\
\text { - haematoma at injecton site (1) } \\
\text { - itching at injecton site (1) } \\
\text { - nodules at injecton site (1) } \\
\text { - reaction at injecton site (1) } \\
\text { - hypersensitivity at injecton site (1) } \\
\text { - urticaria at injecton site (1) } \\
\text { - hardening at injecton site (1) } \\
\text { - warming at injecton site (1) }\end{array}$ & 24 & $35.8 \%$ \\
\hline recurrence of MS & 6 & $9.0 \%$ \\
\hline sensation of heat & 5 & $7.5 \%$ \\
\hline dyspnoe & 4 & $6.0 \%$ \\
\hline
\end{tabular}
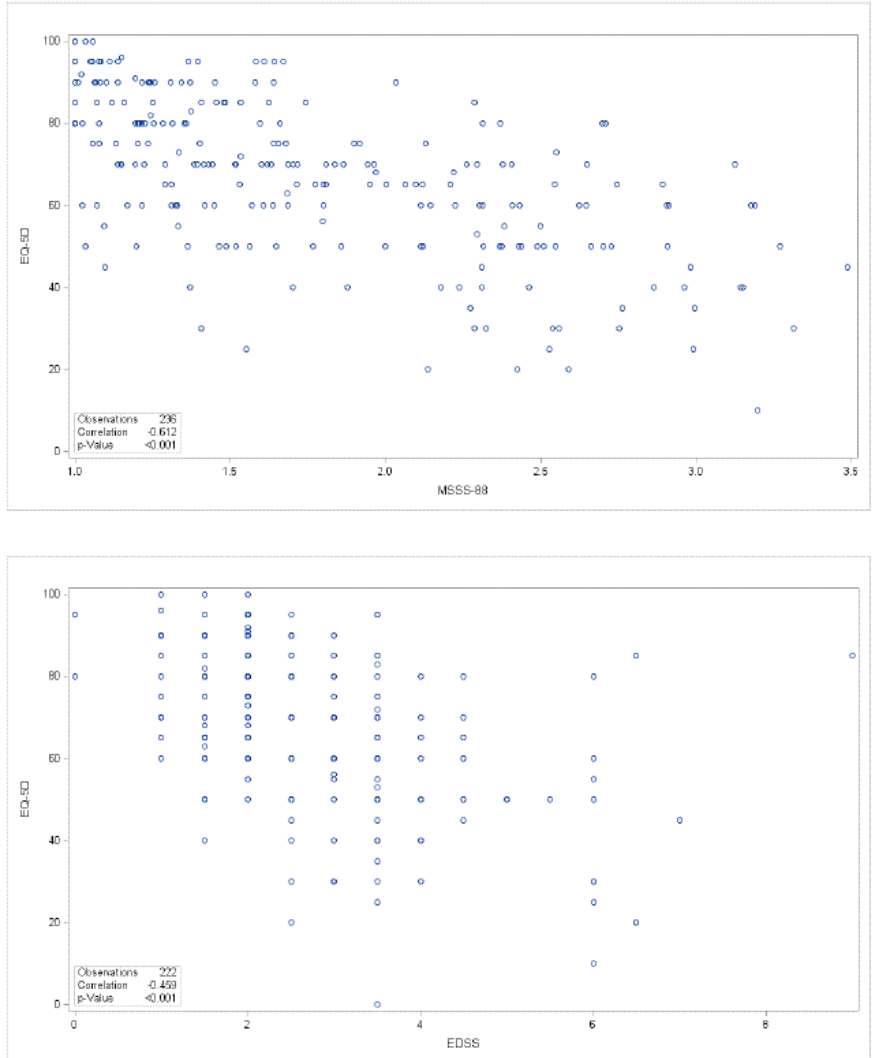

Figure 7. Regression analysis for correlations between MSSS-88 and EQ-5D/VAS and with EDSS. 
Haupts M (2018) Efficacy, tolerability, quality of life and spasticity in outpatients with remitting multiple sclerosis (RMS) treated with glatiramer acetate (GA) Results of an observational study

even rather seldom events is increased - an important aspect for drug safety.

In this non-interventional study with glatiramer acetate the treatment of 305 patients suffering from (mostly remitting-relapsing) multiple sclerosis was documented over a period of nearly one year under daily practice conditions. The daily dose of GA was $20 \mathrm{mg} / \mathrm{ml}$. About two thirds of the patients $(68.1 \%)$ were "de novo" patients. Other patients switched to GA from interferone treatment - mainly due to side effects or lack of efficacy of this pretreatment.

The annualised relapse rate did not change during the study. However, the percentage of patients without relapse during the study improved significantly from $66.5 \%$ to $76.2 \%$ in the entire cohort $(\mathrm{n}=248, \mathrm{p}<0.0114)$, from $63.4 \%$ to $77.6 \%$ in de-novo patients $(\mathrm{n}=161$, $\mathrm{p}<0.0043)$ and not significantly from $67.2 \%$ to $71.6 \%(\mathrm{n}=67, \mathrm{p}=0.5127)$ in patients who switched to GA therapy.

Mean EDSS remained unchanged at $2.7( \pm 1.3)$ points. $13.6 \%$ of the patients showed a progression.

Progression of the disease as measured by ARR and EDSS was stabilized in a majority of patients. Formal clinical NEDA-2 over 1 year was $64.77 \%$. This rate compares to data from other studies as e.g. a oneyear NEDA-3 of $20,4 \%$ in the Avonex-Steroids- Azathioprine (ASA) trial [15].

The mean Ashworth score improved from $1.2( \pm 0.4)$ to $1.12( \pm$ $0.5)$ during the course of the study. $22 \%$ of the patients showed an "improvement" on this scale. $66 \%$ remained unchanged.

The self-reported patient's health status improved or remained stable in all dimensions of EQ-5D in about $80 \%$ of them.

The mean total score of the multiple sclerosis spasticity scale (MSSS-88) remained stable. Subscale improvements were most pronounced in emotional health (46.5\%), pain/discomfort (41.9\%) and muscle stiffness (41.1\%).

Thus there was no progression of spasticity symptoms as assessed by Ashworth Scale and MSSS- 88 .

Patient reported outcome measures (PRO) proved to be valuable assessments: Multiples sclerosis spasticity scale (MSSS-88) and EuroQuol questionnaire (EQ-5D) yielded consistent results with significant correlations. This finding compares well to other studies (3) and adds data from pharmacological treatments im MS.

MSSS-88 proved its value as a novel measurement instrument (tool) to quantify spasticity in spasticity, which is more easy to use in daily clinical practice compared to established scores to measure spastic symptoms in MS. It is easier to use for statistical calculation and more feasible use for long term observation of spastic symptoms. It showed good correlations to QoL as assessed with EQ-5D-VAS, so the severity level measured by MSSS- 88 provides comparable results to longer established instruments.

The tolerability of GA was rated "very good" or "good" in $>80 \%$ of the patients. Adverse events were reported by $9.8 \%$ of the patients, mainly injection site reactions.
It could be shown under everyday clinical practice conditions and based on real life data that the one year treatment with GA had a positive effect on many symptoms of MS, including spasticity, and thus to an improvement of quality of life. GA has shown to be effective and safe either in inital therapy of MS as well as switch treatment after interferone therapy.

These results are consistent with the pivotal GA studies [12] and a former observational study [5] confirming that GA is an efficacious and well tolerated treatment option for MS patients in every stage of disease.

\section{References}

1. Beard S, Hunn A, Wight J (2003) Treatments for spasticity and pain in multiple sclerosis: a systematic review. Health Technol Assess 7: 1-111. [Crossref]

2. Rizzo MA, Hadjimichael OC, Preiningerova J, Vollmer TL (2004) Prevalence and treatment of spasticity reported by multiple sclerosis patients. Mult Scler 10: 589-595. [Crossref]

3. Milinis K, Tennant A, Young CA, TONiC study group (2016) Spasticity in multiple sclerosis: Associations with impairments and overall quality of life. Mult Scler Relat Disord 5: 34-39. [Crossref]

4. Ford C, Goodman AD, Johnson K, Kachuck N, Lindsey JW, et al. (2010) Continuous long-term immunomodulatory therapy in relapsing multiple sclerosis: results from the 15-year analysis of the US prospective open-label study of glatiramer acetate. Mult Scler 16: 342-350. [Crossref]

5. Ziemssen T, Calabrese P, Penner IK, Apfel R (2016) QualiCOP: real-world effectiveness, tolerability, and quality of life in patients with relapsing-remitting multiple sclerosis treated with glatiramer acetate, treatment-naïve patients, and previously treated patients. J Neurol 263: 784-791. [Crossref]

6. Conner (2015) Chapter on Copaxone in the era of biosimilars and nanosimilars.

7. Hobart JC, Riazi A, Thompson AJ, Styles IM, Ingram W, et al. (2006) Getting the measure of spasticity in multiple sclerosis: the Multiple Sclerosis Spasticity Scale (MSSS-88). Brain 129: 224-234. [Crossref]

8. Meca-Lallana JE, Balseiro JJ, Lacruz F, Guijarro C, Sanchez O, et al. (2012) Spasticity improvement in patients with relapsing-remitting multiple sclerosis switching from interferon-I $\hat{I}^{2}$ to glatiramer acetate: the Escala Study. J Neurol Sci 315: 123-128. [Crossref]

9. Kurtzke JF (1983) Rating neurologic impairment in multiple sclerosis: an expanded disability status scale (EDSS). Neurology 33: 1444-1452. [Crossref]

10. Bohannon R, Smith M (1987) Interrater reliability of a modified Ashworth scale of muscle spasticity. Physical Therapy 67: 206-207. [Crossref]

11. Brooks R, Rabin R, de Charro F (2003) The Measurement and Valuation of Health Status Using EQ-5D: A European Perspective: Evidence from the EuroQol BIO MED Research Programme. Rotterdam: Kluwer Academic Publishers.

12. Bornstein MB, Miller A, Slagle S, Weitzman M, Crystal H, et al. (1987) A pilot trial of Cop 1 in exacerbating-remitting multiple sclerosis. $N$ Engl J Med 317: 408-414. [Crossref]

13. EMA/CHMP/771815/2011, Rev. 2: Guideline on clinical investigation of medicinal products for the treatment of Multiple Sclerosis. 26 March 2015.

14. Polman CH1, Reingold SC, Banwell B, Clanet M, Cohen JA, et al. (2011) Diagnostic criteria for multiple sclerosis: 2010 revisions to the McDonald criteria. Ann Neurol 69 292-302. [Crossref]

15. Havrdova E, Zivadinov R, Krasensky J, Dwyer MG, Novakova I, et al. (2009) Randomized study of interferon beta-1a, low-dose azathioprine, and low-dose corticosteroids in multiple sclerosis. Mult Scler 15: 965-976. [Crossref]

Copyright: (C2018 Haupts M. This is an open-access article distributed under the terms of the Creative Commons Attribution License, which permits unrestricted use, distribution, and reproduction in any medium, provided the original author and source are credited. 